\title{
Molecular Weight Distribution of a Segmented Copolymer of PET-PCL
}

\author{
WOO KA FAI, ${ }^{1}$ WU CHI, ${ }^{1, *}$ MA DE ZHU, ${ }^{2}$ and LUO XIAO LIE ${ }^{2}$ \\ ${ }^{1}$ Department of Chemistry, The Chinese University of Hong Kong, Shatin, N.T., Hong Kong, and ${ }^{2}$ Department of \\ Materials Science and Engineering, The University of Science \& Technology of China, Hefei, Anhui, China
}

\begin{abstract}
SYNOPSIS
A recently developed method of combining both the results of static and dynamic laser light scattering (LLS) was applied to characterize the segmented copolymer of poly(ethylene terephthalate-co-caprolactone) (PET-PCL) with $48 \%$ PET content. Unlike the traditional three-solvent method proposed by Bushuk and Benoit for copolymers, this method requires only two solvents. For each solvent, the apparent weight average molecular weight $\left(M_{w, \text { app }}\right)$ was measured by static LLS. Then $M_{w, \text { app }}$ was used as a constraint to convert the linewidth distribution from dynamic LLS into the apparent molecular weight distribution. Finally, the two apparent molecular weight distributions were combined to give both the true molecular weight distribution and the estimated chain composition distribution of the copolymer. We found that the PET-PCL sample with $48 \%$ PET is nearly uniform in the chain composition. (c) 1995 John Wiley \& Sons, Inc.
\end{abstract}

\section{INTRODUCTION}

The segmented copolymer of poly (ethylene terephthalate-co-caprolactone) (PET-PCL) is a thermal viscoelastic material. The hard segments (PET) can crystallize to form the physical crosslinking points and the soft segments (PCL) attribute to the elasticity of the materials. As the amount of hard segments increases, this segmented copolymer can gradually change from a low module elastomer to a high module tough plastic. Therefore, the characterization of this type of segmented copolymer, especially its absolute molecular weight and chain composition distribution, is crucially important to its various applications.

Traditionally, the weight average molecular weight ( $M_{w}$ ) of a copolymer can be determined by using the Bushuk-Benoit light scattering theory. ${ }^{1}$ According to this theory, at least three solvents in which the copolymer has different refractive index increments $(\nu)$ are required. However, the choice of three such solvents is usually very difficult because they have to simultaneously satisfy the following

\footnotetext{
* To whom correspondence should be addressed. Journal of Applied Polymer Science, Vol. 57, 1285-1290 (1995) (C) 1995 John Wiley \& Sons, Inc. CCC $0021-8995 / 95 / 111285-06$
}

conditions: first, the $\nu$ values of three solvents should be as different as possible; second, copolymer should be soluble in these solvents; third, the copolymer solution should have as little as possible absorption at the incident laser light wavelength; and last, but not least, the solution clarification, such as the removing of dust, should not be extremely difficult. Owing to the difficulty in selecting such three solvents, a modified laser light scattering (LLS) method of requiring only two solvents was proposed to determine the copolymer true molecular weight. ${ }^{2}$ This work shows another application of this new method.

\section{THEORY}

\section{Static Light Scattering}

The angular dependence of the excess absolute timeaveraged scattered intensity, known as the excess Rayleigh ratio, $R_{v v}(\theta)$, of a dilute polymer solution at concentration $C(\mathrm{~g} / \mathrm{mL})$ and scattering angle $\theta$ can be expressed as $^{3}$ 


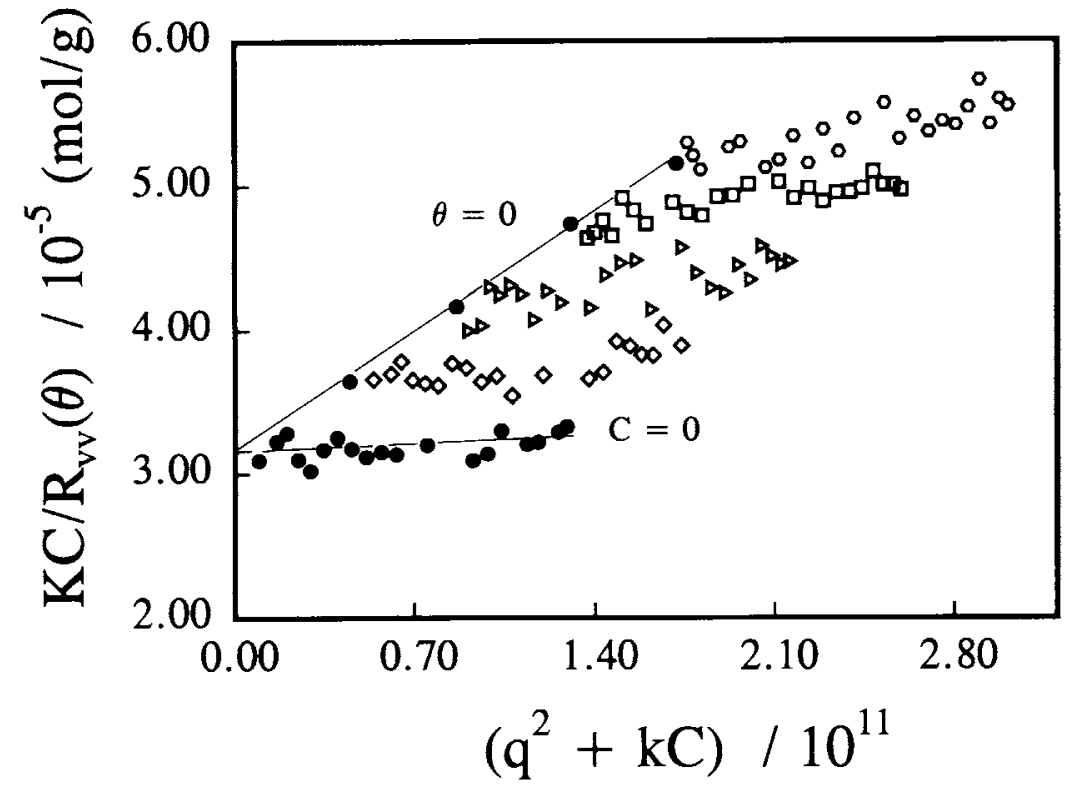

Figure 1 Typical static Zimm plot of the 48\% PET PET-PCL segmented copolymer in chloroform at $25^{\circ} \mathrm{C}$. The concentration range is from $1.03 \times 10^{-3}$ to 3.95 $\times 10^{-3} \mathrm{~g} / \mathrm{mL}$.

$$
\frac{K C}{R_{v v}(\theta)} \approx \frac{1}{M_{w} P(\theta)}+2 A_{2} C
$$

where $K=4 \pi^{2} n^{2} \nu^{2} /\left(N_{A} \lambda_{0}^{4}\right)$ with $N_{A}, n, \lambda_{0}$, and $\nu$ being Avogadro's number, the solvent refractive index, the wavelength of light in vacuo, and the refractive index increment $(d n / d C)$, respectively. At $\left\langle R_{g}^{2}\right\rangle_{2} q^{2}<1,1 / P(\theta) \approx 1+\frac{1}{3}\left\langle R_{g}^{2}\right\rangle_{2} q^{2}$ where $\left\langle R_{g}^{2}\right\rangle Z^{1}{ }^{2}$ is root-mean-square $z$-average radius; and $q\left(=4 \pi n / \lambda_{0} \sin (\theta / 2)\right)$ is the scattering vector. By measuring $R_{v v}(\theta)$ at a set of $C$ and $\theta$, we can determine $M_{w},\left\langle R_{g}^{2}\right\rangle Z^{1 / 2}$, and $A_{2}$ from a Zimm plot that incorporates $\theta$ and $C$ extrapolations on a single grid.

For a polydisperse copolymer with both molecular weight and chain-composition distributions, $M_{w}$ determined on the basis of eq. (1) will only be an apparent weight average molecular weight $M_{w, a p p}$, defined as ${ }^{1}$

$$
M_{w, \mathrm{app}}=\int_{0}^{\infty} f_{w}(M)\left(\frac{\nu(M)}{\nu}\right)^{2} M d M
$$

where $f_{w}(M)$ is the true molecular weight distribution and $\nu(M)$ is the refractive index increment for molecules with molecular weight $M$ and weight distribution $f_{w}(M) . f_{w}(M)(\nu(M) / \nu)^{2}$ is defined as the apparent weight distribution $f_{w, \text { app }}(M)$ hereafter. On the basis of the additivity assumption of $\nu$ for a segmented copolymer composed of monomeric units $A$ and $B^{1}$ (in this study, $A$ is ET and $B$ is CL),

$$
\nu(M)=W_{A}(M) \nu_{A}+W_{B}(M) \nu_{B}
$$

and

$$
\nu=W_{A} \nu_{A}+W_{B} \nu_{B}
$$

Table I Summary of Static and Dynamic LLS Results of 48\% PET PET-PCL Segmented Copolymer in $\mathrm{CHCl}_{3}$ and $\mathrm{THF}$ at $25^{\circ} \mathrm{C}$

\begin{tabular}{lccccccc}
\hline \multicolumn{1}{c}{ Solvent } & $\begin{array}{c}10 \nu^{\mathrm{a}} \\
(\mathrm{mL} / \mathrm{g})\end{array}$ & $\begin{array}{c}10^{-4} M_{w, \mathrm{app}} \\
(\mathrm{g} / \mathrm{mol})\end{array}$ & $\begin{array}{c}10^{3} A_{2} \\
\left(\mathrm{~mol} \mathrm{~mL} / \mathrm{g}^{2}\right)\end{array}$ & $\begin{array}{c}\left\langle R_{g}^{2}\right\rangle Z^{1 / 2} \\
(\mathrm{~nm})\end{array}$ & $\begin{array}{c}10^{7} \bar{D} \\
\left(\mathrm{~cm}^{2} / \mathrm{s}\right)\end{array}$ & $\alpha_{D}$ & $\begin{array}{c}10^{4} k_{D} \\
(\mathrm{~g} / \mathrm{mol})\end{array}$ \\
\hline Chloroform & 1.00 & 3.22 & 2.5 & $<10$ & 5.85 & 0.61 & 3.64 \\
Tetrahydrofuran & 1.20 & 3.33 & 0.45 & $<10$ & 7.09 & 0.61 & 4.52 \\
\hline
\end{tabular}

a The relative uncertainty of measured $\Delta n$ is about $\pm 1 \%$. 


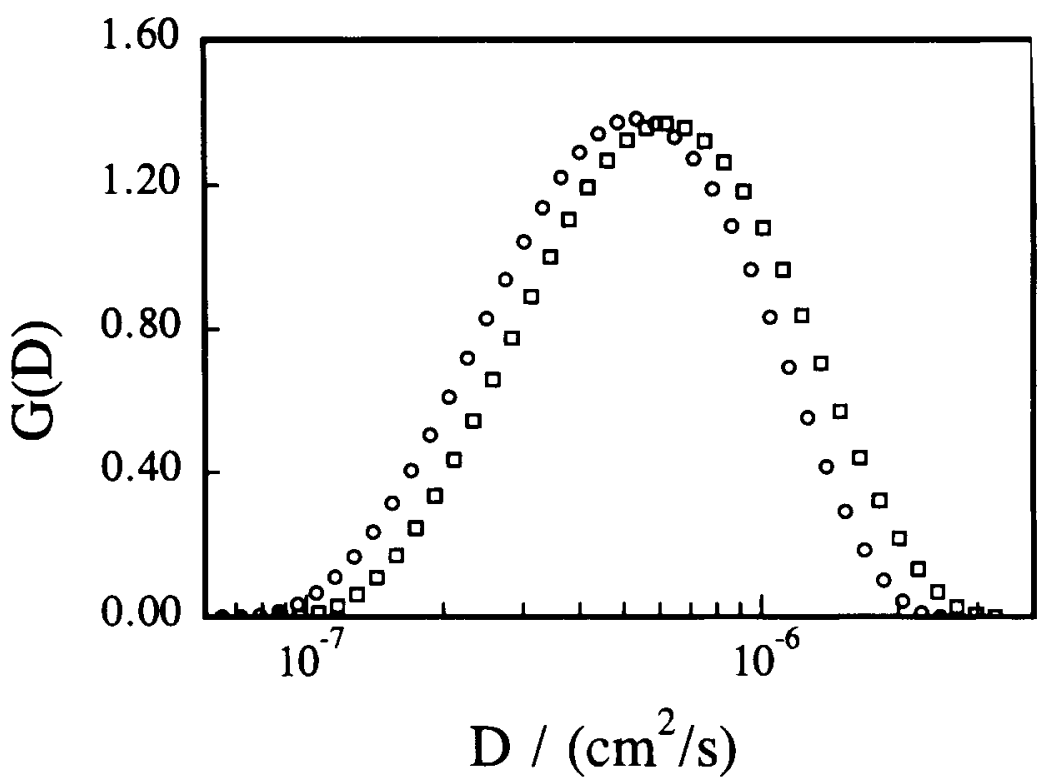

Figure 2 Translational diffusion coefficient distributions of the 48\% PET PET-PCL segmented copolymer $(\mathrm{O})$ in $\mathrm{CHCl}_{3}$ and $(\square)$ in $\mathrm{THF}$ at $25^{\circ} \mathrm{C}$.

where $W_{A}(M)$ and $W_{B}(M)\left[\equiv 1-W_{A}(M)\right]$ are the weight fractions (chain composition) of $A$ and $B$ at a given $M$ and $f_{w}(M)$, respectively, and $W_{A}$ and $W_{B}$, the overall weight fractions (chain composition) of $A$ and $B$, respectively. $W_{A}$ and $W_{B}$ are usually known parameters from polymerization. For a chosen solvent, $\nu_{A}, \nu_{B}$ and $\nu$ are constants for a given copolymer at fixed experimental conditions. It is obvious that for a uniform chain composition, that is, $W_{A}(M)$ $=W_{A}, W_{B}(M)=W_{B}$, and $\nu(M)=\nu$, we have $M_{w \text {,app }}$ $=M_{W}$. It should be noted that eq. (3) is not unique because different chain compositions can give a polymer chain with the identical $M$ and $f_{w}(M)$.

\section{Dynamic Light Scattering}

In dynamic LLS, a precise intensity-intensity time correlation function $G^{(2)}(t, \theta)$ in the self-beating mode can be measured that has the following form ${ }^{4,5}$

$$
G^{(2)}(t, \theta)=\langle I(t, \theta) I(0, \theta)\rangle=A\left[1+\beta\left|g^{(1)}(t, \theta)\right|^{2}\right]
$$

where $A$ is a measured baseline, $\beta$ is a parameter depending on the coherence of the detection, $t$ is the delay time, and $g^{(1)}(t, \theta)$ is the normalized first-order electric field time correlation function. For a polydisperse sample, $g^{(1)}(t, \theta)$ is related to the line-width distribution $G(\Gamma)$ by

$$
g^{(1)}(t, \theta)=\left\langle E(t, \theta) E^{*}(0, \theta)\right\rangle=\int_{0}^{\infty} G(\Gamma) e^{-\Gamma t} d \Gamma .
$$

The mostly accepted Laplace inversion analysis program CONTIN ${ }^{6}$ was used in the present work to convert the measured $G^{(2)}(t, \theta)$ to $G(\Gamma)$. The line width $\Gamma$ usually depends on both $C$ and $\theta$. This dependence can be expressed as

$$
\frac{\Gamma}{q^{2}}=D\left(1+k_{d} C\right)\left(1+f\left\langle R_{g}^{2}\right\rangle_{Z} q^{2}\right)
$$

where $D$ is the translational diffusion coefficient at $C=0$ and $q=0, f$ is a dimensionless number, and $k_{d}$ is the diffusion second virial coefficient. The value of $f$ depends on the chain structure, polydispersity, and solvent power.

From eqs. (1), (2), and (6), at the conditions of $C$ $\rightarrow 0, \theta \rightarrow 0$, and $t \rightarrow 0$, we have

$$
R_{v v}(\theta)=K C \int_{0}^{\infty} f_{w}(M) M\left(\frac{\nu(M)}{\nu}\right)^{2} d M \propto\langle I\rangle
$$

and

$$
\begin{aligned}
g^{(1)}(0, \theta) & =\left\langle E(0, \theta) E^{*}(0, \theta)\right\rangle \\
& =\int_{0}^{\infty} G(\Gamma) d \Gamma \propto\langle I\rangle
\end{aligned}
$$

that leads to

$$
\int_{0}^{\infty} G(D) d D=\gamma \cdot \int_{0}^{\infty} f_{w}(M) M\left(\frac{\nu(M)}{\nu}\right)^{2} d M
$$




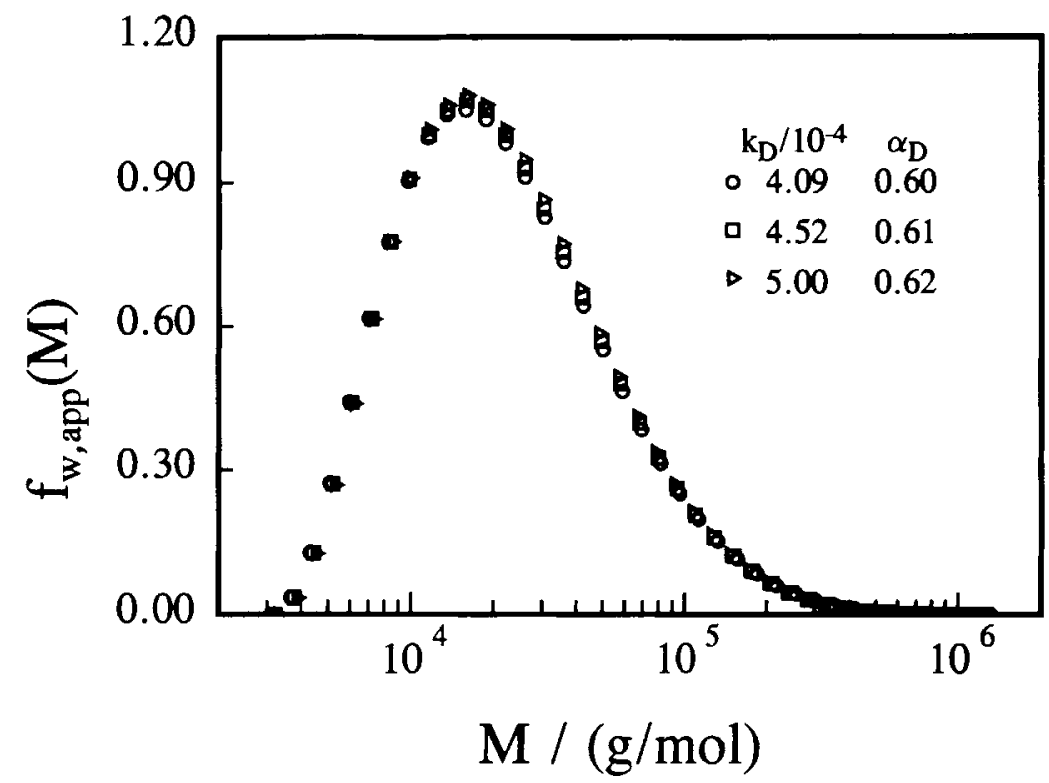

Figure 3 Comparison of three apparent molecular weight distributions of the $48 \%$ PET PET-PCL segmented copolymer in THF, calculated using eqs. (11) and (13), with three different pairs of $k_{D}$ and $\alpha_{D}$.

where $\gamma$ is a normalization constant. Normally, $D$ can be related to $M$ by using two scaling constants, $k_{D}$ and $\alpha_{D}$, that is

$$
D=k_{D} M^{-\alpha_{D}}
$$

where both $k_{D}$ and $\alpha_{D}$ are independent on $M$ for a given solvent and chain composition in given experimental conditions. Using eq. (11), we can rewritten eq. (10) as

$$
\begin{aligned}
\int_{0}^{\infty} G(D) \frac{d D}{d M} d M & \\
& =\gamma \cdot \int_{0}^{\infty} f_{w}(M) M\left(\frac{\nu(M)}{\nu}\right)^{2} d M .
\end{aligned}
$$

After comparing both sides of eq. (12), we have

$$
f_{w, \mathrm{app}}(M)=f_{w}(M)\left(\frac{\nu(M)}{\nu}\right)^{2} \propto \frac{G(D)}{M} \cdot \frac{d D}{d M}
$$

where all proportional constants have been omitted because they are irrelevant to both distributions. For a given set of $k_{D}$ and $\alpha_{D}$, we are able to convert $G(D)$ into $f_{w, \text { app }}(M)$ by using eqs. (11) and (13).

As stated by Chu et al., ${ }^{7}$ if the above procedure was repeated by using two solvents ( 1 and $\mathrm{s} 2$ ) with different $\nu(M)$ and $\nu$, we will have two different apparent weight distributions, $f_{w, \text { app }, \mathrm{s} 1}(M)$ $=f_{w}(M)[\nu(M) / \nu]_{\mathrm{s} 1}^{2}$ and $f_{w, \mathrm{app}, \mathrm{s} 2}(M)=f_{w}(M)[\nu(M) / \nu]_{\mathrm{s} 2}^{2}$ for a given copolymer sample. With the substitution of eq. (3), the ratio of two apparent weight distributions will be

$$
\begin{aligned}
& \frac{f_{w, \mathrm{app}, \mathrm{s} 1}(M)}{f_{w, \mathrm{app}, \mathrm{s} 2}(M)} \\
& \quad=\left\{\frac{\nu_{\mathrm{s} 2}}{\nu_{\mathrm{s} 1}} \cdot \frac{W_{A}(M) \nu_{A, \mathrm{~s} 1}+\left[1-W_{A}(M)\right] \nu_{B, \mathrm{~s} 1}}{W_{A}(M) \nu_{A, \mathrm{~s} 2}+\left[1-W_{A}(M)\right] \nu_{B, \mathrm{~s} 2}}\right\}^{2}
\end{aligned}
$$

where $v_{\mathrm{s} 1}, \nu_{\mathrm{s} 2}, \nu_{A, \mathrm{~s} 1}, \nu_{A, \mathrm{~s} 2}, v_{B, \mathrm{~s} 1}$, and $\nu_{B, \mathrm{~s} 2}$ can be separately determined by a differential refractometer. ${ }^{8}$ Hence we can solve eq. (14) to get $W_{A}(M)$, the chaincomposition distribution of polymer $A$, from two apparent weight distributions. After having $W_{A}(M)$, we are ready to calculate $\nu(M)$ [by eq. (3)], $f_{w}(M)$ (by definition of $f_{w, \text { app }}$ ), and $M_{w}$ (by definition of $M_{W}$ ).

\section{EXPERIMENTAL}

\section{Sample}

The synthesis of the segmented copolymer of PETPCL was performed in two separate steps: ${ }^{9}$ step I involves the esterification of terephthalic acid and ethylene glycol in the present of a catalyst at $190^{\circ} \mathrm{C}$; and step II is a polycondensation of ET with CL in the presence of a catalyst under vacuum at $250^{\circ} \mathrm{C}$. The content of PET in the copolymer is $48 \%$ by weight. 


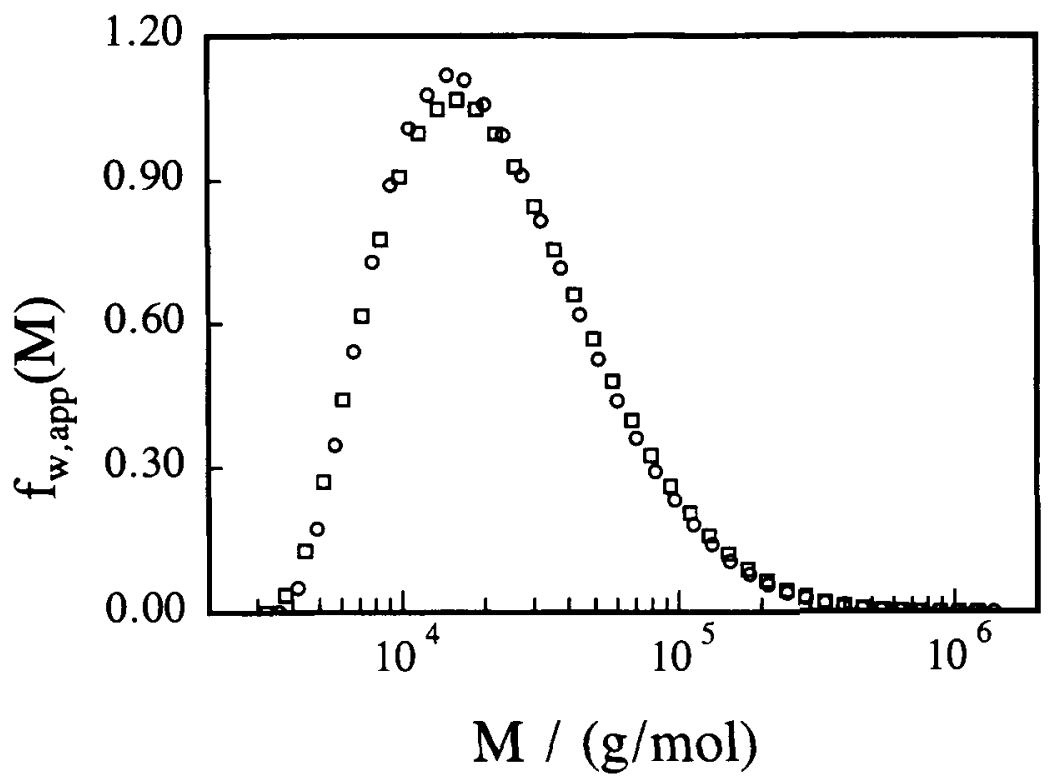

Figure 4 Apparent molecular weight distributions of the $48 \%$ PET PET-PCL segmented copolymer $(\mathrm{O})$ in $\mathrm{CHCl}_{3}$ and $(\square)$ in THF calculated from the distributions in Figure 2.

\section{Solution Preparation}

Two analytical grade solvents (Merck; chloroform, $\mathrm{CHCl}_{3}$, and tetrahydrofuran, THF) were used without further purification. The solution concentrations were in the range $1 \times 10^{-3}$ to $6 \times 10^{-3} \mathrm{~g} / \mathrm{mL}$. All polymer solutions were clarified at room temperature by using a $0.22-\mu \mathrm{m}$ Millipore $\left(\right.$ Millex- $\mathrm{GV}_{13}$ ) filter to remove dust.

\section{LLS}

A commercial LLS spectrometer (ALV DLS/SLS5000 , Langen, Hessen, Germany) was used with an argon ion laser (Coherent INNOVA 90, operated at wavelength $488 \mathrm{~nm}$ and $100 \mathrm{~mW}$ ) as the light source. The primary beam is vertically polarized. All refractive index increments were determined by using a novel differential refractometer. ${ }^{10}$ The intensity-intensity time correlation functions was measured by an ALV 5000 multiple tau digital correlator. The detail of LLS instrumentation can be found elsewhere. ${ }^{5}$ All measurements were done at $25.0 \pm 0.2^{\circ} \mathrm{C}$.

\section{RESULTS AND DISCUSSION}

Figure 1 shows a typical Zimm plot of $48 \%$ PET in chloroform. On the basis of eq. (1), we can calculate $M_{w, \text { app }}, A_{2}$, and $\left\langle R_{g}^{2}\right\rangle^{1 / 2}$ from the extrapo- lation of $\left[K C / R_{v v}(\theta)\right]_{\theta \rightarrow 0, C \rightarrow 0}$ and $\left[K C / R_{v v}(\theta)\right]_{\theta \rightarrow 0}$ vs. $C$ and $\left[K C / R_{v v}(\theta)\right]_{C \rightarrow 0}$ vs. $q^{2}$, respectively. The results are summarized in Table $\mathrm{I}$. We should note that the molecular sizes of $48 \%$ PET are so small that the scattering intensities are virtually independent of the scattering angle. Therefore, it is impossible to determine the exact values of $\left.\left\langle R_{g}^{2}\right\rangle\right\rangle^{1 / 2}$. The positive values of $A_{2}$ indicate that both $\mathrm{CHCl}_{3}$ and THF are good solvents for the PET-PCL sample at $25^{\circ} \mathrm{C}$.

Figure 2 shows two translational diffusion coefficient distributions of $48 \%$ PET in chloroform and tetrahydrofuran at $25^{\circ} \mathrm{C}$. In the process of reducing $G(\Gamma)$ to $G(D)$, we have used $\bar{f}$ and $\bar{k}_{d}$ in Wu et al. ${ }^{2}$ to get the $G(D)$ value at $\theta \rightarrow 0$ and $C$ $\rightarrow 0$. On the basis of eq. (7), we can see that the correction of $\left(1+\bar{k}_{d} C\right)\left(1+\bar{f}\left\langle R_{g}^{2}\right\rangle_{z} q^{2}\right)$ are a few percent. The values of average $\bar{D}$ in two solvents are listed in Table I. $G(D)$ in Figure 2 can be converted to $f_{w \text {,app }}(M)$ for a given pair of $\alpha_{D}$ and $k_{D}$. From the previous study, ${ }^{2}$ we know that $\alpha_{D}=0.61$ \pm 0.01 for a $58 \%$ PET sample in $\mathrm{CHCl}_{3}$ and THF. Because $\alpha_{D}$ is only related to the polymer chain conformation in solution, we have assumed that for the $48 \%$ PET sample $\alpha_{D}$ remained unchanged.

Figure 3 shows that a slight mischoice of $\alpha_{D}$ has no significant effect on the final apparent molecular weight distribution. With this $\alpha_{D}$ we can determine $k_{D}$ from $M_{w, \text { app }}$ and $G(D)$ by using the following equation ${ }^{11-13}$ 


$$
M_{w, \text { app }}=\frac{k_{D}^{1 / \alpha_{D}} \int_{0}^{\infty} G(D) d D}{\int_{0}^{\infty} G(D) D^{1 / \alpha_{\mathrm{D}}} d D}
$$

The values of $k_{D}$ and $\alpha_{D}$ for $48 \%$ PET sample in two solvents are listed in Table I. After having $k_{D}$ and $\alpha_{D}$, we are ready to transfer $G(D)$ in Figure 2 into $f_{w, \text { app }}(M)$ by eqs. (11) and (13).

Figure 4 shows two apparent molecular weight distributions based on two diffusion coefficient distributions in Figure 2. One interesting result in Figure 4 is that the two apparent weight distributions are almost identical if we consider the uncertainties in the experiment. This suggested that the chain composition $\left[W_{\mathrm{PET}}(M)\right.$ and $\left.W_{\mathrm{PCL}}(M)\right]$ are nearly independent of $M$. Therefore, $f_{w, \text { app }}(M) \mathrm{s}$ in Figure 4 represent an approximation of the true molecular weight distribution $f_{w}(M)$. The $M_{w} / M_{n}$ calculated from $f_{w}(M)$ is $\sim 2.1$ that is slightly greater than 2.0 predicted by the polycondensation reaction kinetics. This might be due to the uncertainties introduced in both the experiment and the data analysis.

\section{CONCLUSION}

The molecular weight distribution of the PET-PCL segmented copolymer with $48 \%$ PET content was characterized by a combination of static and dynamic LLS where only two solvents were used. We found that the chain composition of this segmented copolymer is nearly independent of its molecular weight, so that the apparent molecular weight distributions in $\mathrm{CHCl}_{3}$ and THF can be treated as the true molecular weight distribution. The successful application of this two-solvent LLS method to the 48\% PET sample further shows that the following procedure can be used as a general method to characterize segmented copolymers. In this two-solvent
LLS method, one can first determine the apparent molecular weight $M_{w}$ in two solvents from static LLS, then two apparent weight distributions $f_{w \text {,app }}(M)$ from dynamic LLS. Further, a ratio of these two $f_{w \text {,app }}(M) \mathrm{s}$ at each $M$ will lead to the chain composition $W_{A}(M)$. Finally, the true molecular weight distribution $f_{w}(M)$ can be calculated from $f_{w, \text { app }}(M)$ and $W_{A}(M)$.

The financial support of this work by the RGC (the Research Grants Council of Hong Kong Government) Earmarked Grant 1993/94 (CUHK 79/93E, 221600140) is gratefully acknowledged.

\section{REFERENCES}

1. W. Bushuk and H. Benoit, Can. J. Chem., 36, 1616 (1958).

2. C. Wu, K. F. Woo, X. L. Luo, and D. Z. Ma, Macromolecules, 27 (12), 6055 (1994).

3. B. H. Zimm, J. Chem. Phys., 16(12), 1099 (1948).

4. R. Pecora and B. J. Berne, Dynamic Light Scattering, Plenum Press, New York, 1976.

5. B. Chu, Laser Light Scattering, Academic Press, New York, 1974.

6. S. W. Provencher, Biophys. J., 16, 27 (1976); J. Chem. Phys., 64(7), 2772 (1976).

7. B. Chu, Q. Ying, D. C. Lee, and D. Q. Wu, Macromolecules, 18, 1962 (1985).

8. C. Wu, D. Z. Ma, X. L. Luo, et al., J. Appl. Polym. Sci., 53, 1323 (1994).

9. X. L. Luo, D. Z. Ma, and D. Z. Du, J. Univ. Sci. Technol. China, 16 (3), 280 (1986).

10. C. Wu and K. Q. Xia, Rev. Sci. Instrum., 65(3), 587 (1994).

11. C. Wu and D. Lilge, J. Appl. Polym. Sci., 50, 1753 (1993).

12. C. Wu, Macromolecules, 26, 3821 (1993).

13. C. Wu, Macromolecules, 26, 5423 (1993).

Received October 31, 1994

Accepted March 6, 1995 